\title{
Assessment of Tree Diversity in Agroforestry Systems under Irrigated Ecosystem
}

\author{
Doddabasawa $^{1^{*}}$, M. Mahadeva Murthy ${ }^{1}$ and B.M. Chittapur ${ }^{2}$ \\ ${ }^{1}$ Department of Forestry and Environmental Science, UAS, GKVK, \\ Bangaluru-65, Karnataka, India \\ ${ }^{2}$ Chief Scientific Officer, UAS, Raichur, Karnataka, India \\ *Corresponding author
}

\begin{tabular}{l} 
K e y w o r d s \\
$\begin{array}{l}\text { Agroforestry, Bund } \\
\text { planting, Species } \\
\text { density, Species } \\
\text { richness, Silvihorti. }\end{array}$ \\
Article Info \\
$\begin{array}{l}\text { Accepted: } \\
\text { 26 September } 2017 \\
\text { Available Online: } \\
\text { 10 October } 2017\end{array}$ \\
\hline
\end{tabular}

Status of agroforestry systems and assessment of tree diversity in the existing agroforestry systems of North-eastern part of Karnataka under irrigated ecosystem was carried out during 2015-16. There were six prominent agroforestry systems practiced by the farmers and the majority farmers practiced bund planting $(43.06 \%)$ followed by boundary planting (19.44\%), silvihorti systems (12.50\%), scattered planting (4.17\%) and silvipasture system $(2.78 \%)$ while block plantation system was found to be associated with one or other systems on the farm land. 52 tree species were recorded with a mean species density of $9.04 \mathrm{ha}^{-1}$ and mean number of trees were $104.24 \mathrm{ha}^{-1}$. Further, significantly higher number of trees per hectare occurred in silvihorti system (123) followed by boundary planting (105.43) and bund planting (93.10) whereas least number of trees per hectare were recorded in silvipasture system (48.00) followed by scattered planting (69.33). Interestingly, significantly higher Shannon diversity index was observed in scattered planting (1.79) followed by silvipasture system (1.72) whereas lower value was recorded in bund planting (1.04). Among different categories of holding size, higher number of trees per hectare was recorded with large farmer (122.48) followed by medium farmer (100.45); lowest being with small farmer (91.82). Among the districts, significantly higher mean number of species per hectare was observed in Kalaburagi district (11.25) followed by Bidar (9.08) and lower mean number of species per hectare was recorded in Koppal (7.75) followed by Ballari (8.58). Tectona grandis, Azadirachta indica, and Cocus nucifera were the preferred perennials.

\section{Introduction}

Agroforestry systems are multifunctional land use systems where woody perennials are deliberately integrated with the field crops and or livestock rearing under the same unit of land management either at the same time or in sequence. These land use systems provide economical benefits besides extending ecological benefits commonly associated with agroforestry systems and the predominant ones are enhanced soil fertility, improved water quality, and enhanced biodiversity and carbon sequestration (Nair, 2011).

The composition and pattern of agroforestry land use systems are location specific, performance biased, and preference of the farmer and culture of the countries based (Nair et al., 2008). Therefore, the characteristic of the existing agroforestry systems are influenced by the heterogeneous 
factors as agriculture in the tropics is practiced under diversified agro-ecological conditions and within each agro-ecological zone, farm management is rarely homogeneous. Variability also exists within every agro-ecological condition at regional level and at farm level, farm management strategy, economic condition of the farmer, availability of land, etc., (Giller et al., 2006). Other major factors which decide the retention of the tree species on the farm land by the farmer are defined to meet their multiple and diverse needs mainly the utility, economical product, and compatibility and adoptability of species. Therefore, the agroforestry land use systems are highly complex in nature. Hence, agroforestry systems need to be studied with multidimensional approach with socioeconomic and ecological prospective view (Tittonell et al., 2005). However, it is concerning to mention that despite significance of trees in biodiversity and ecological security, due to intensification of grain based agriculture for short term economic reasons, the traditional agroforestry systems are disappearing from the landscape (Nerlich, et al., 2013). Therefore, taking stock of the situation to provide sound footing for any future land/and vegetation based strategy. Keeping these points in view, the present study was envisaged to delineate present status of existing agroforestry systems in the north eastern part of Karnataka under irrigated ecosystem.

\section{Materials and Methods}

The study was under taken in North Eastern part of Karnataka, India (comprising north eastern transitional zone (Zone I), north eastern dry zone (Zone II) and northern dry zone of the state (Zone III) to assess the tree diversity in agroforestry systems under irrigated ecosystem. The study area lies within the geographical region of North maiden; it spreads between $14^{0} 60^{\prime}$ to $18^{0} 30^{\prime}$ Northern latitude and $75^{\circ} 60$, to $77^{\circ} 70^{\prime}$ Eastern longitude. The background information of the study area were collected by visiting District statistical office and interacting with staff of line departments and the weather data was collected from the representative meteorological observatories located in the study area. The average annual rainfall ranges from 600 to $900 \mathrm{~mm}$ with an average rainfall of $750 \mathrm{~mm}$ with a $\mathrm{CV}$ over $30 \%$ and mean elevation ranges from 350-680 $\mathrm{m}$ MSL. The soils are deep to very deep black soils and medium black soils in majority areas while sandy loam and light textured soils and laterite soils also found in some pockets. The most of this region is characterized by semiarid to arid climatic condition and covers nearly 23 per cent of total geographical area of the state. The major crops grown are pigeonpea, greengram, bengalgram, groundnut, soybean, sunflower, safflower, sorghum, and pearlmillet, under rainfed condition while cotton, sugarcane and paddy predominate under irrigation.

Multistage purposive randomized sampling technique was used to select the samples for the study by selecting districts as unit (6 districts) and in each district two taluks were identified, in each taluk one village was identified and in each village 6 respondents of 2 each in small farmer ( $<2$ ha), medium farmer ( 2 to $4 \mathrm{ha}$ ) and large farmer (>4ha) were selected randomly among the list of the farmers who are already practicing agroforestry systems. In all, the total sample size of the study was 72 farmers.

The information on the existing traditional agroforestry systems, species richness, diversity and density were recorded by visiting the field physically and interviewing the farmers with structured questionnaire prepared for the study. The kind of agroforestry system practiced by the farmers 
in the study area were identified by visiting the field and classified based on the nature of the component and the pattern of tree planting on the farm land and the number of farmers practicing specific agroforestry systems were recorded and expressed in percentage out of the total farmers surveyed. Species richness, species density and tree density in the existing agroforestry systems were recorded with plot size of one hectare representing the total farm land of each individual farmer.

The data on species richness was obtained by aggregating number of species present and expressed in total number of species per agroforestry systems, per district and per category of farmers. The species density was calculated by aggregating total number of species found in different systems, farmers and per district and expressed as mean number per hectare. Similarly the tree density was calculated by counting total number of trees divided by the number of farmers and expressed as mean number per hectare.

The dominance of the tree species on farm land was calculated by taking the relative density of the species which was calculated by dividing the total number of individual species to the overall total of all the species, and frequency of the species was calculated based the frequency of the occurrence of the species in all the sample plots.

The data on the species diversity was also subjected to Shannon and Simpson's diversity index analyses which was calculated by using Shannon's index $\mathrm{H}^{\prime}=\sum_{i=1}^{n} \mathrm{Pi} \ln \mathrm{Pi}$ and Simpson index $\lambda=\sum_{i=1}^{n} P i^{2}$ where ' $\mathrm{n}$ ' is the total number of species and ' $p$ ' is the relative abundance of $\mathrm{i}^{\text {th }}$ species (christine and Nestor, 2008). The data collected was analyzed for descriptive statistics and one way ANOVA at a significance level of 0.05 and further, to know the difference between the means post hoc test was performed using Duncan test at significance level of 0.05 by using SPSS (Statistical Package for Social Science).

\section{Results and Discussion}

\section{Agroforestry systems practiced by the farmers}

There were six prominent agroforestry systems practiced by the farmers and the majority farmers practiced bund planting (43.06\%) followed by boundary planting (19.44\%), silvihorti systems (12.50\%), scattered planting $(4.17 \%)$ and silvipasture system $(2.78 \%)$ while block plantation system was found to be associated the one or other system on the farm land (Table 1). However, 18.06 per cent of the total respondents had more than one agroforestry systems on their farm land. Whereas only four major systems out of five were practiced by the large farmers except silvi-pasture system which was practiced by small and medium farmers.

Cumulative percentage of the respondents practicing agroforestry systems revealed considerable variation in the practice of agroforestry systems between the categories of the farmers. The majority of respondents among large farmers practiced silvihorticultural system $\quad(42.86 \quad \%$ cumulative) followed by bund planting (38.09 $\%$ cumulative) and boundary planting (14.29 $\%$ cumulative) whereas the bund planting (65.47 and $49.29 \%$ cumulative, respectively) was the major system practiced by medium and small farmers followed by boundary planting $(20.46, \quad 31.82 \%$ cumulative, respectively) and silvihorticultural system (10.34 and $31.82 \%$ cumulative, respectively (Table 1).

Among the districts, bund planting (41.67 to $75.00 \%$ cumulative) was the major practice followed by boundary planting (16.67 to $75.00 \%$ cumulative) and silvihorti system 
(8.33 to $16.67 \%$ cumulative) in Bidar, Kalburagi and Raichur districts. Whereas, in Yadagir and Ballari districts bund planting $(50.00 \%)$ was the major practice followed by silvihorti system $(25.00 \%)$ and boundary planting (16.67 \%). Interestingly, it was silvihorti system which was dominating in Koppal district (41.66\% cumulative) followed by bund planting $(33.33 \%)$ and boundary planting (16.67\%). Thus, agroforestry prevailing in each district revealed differential preference of farmers. Overall, the bund planting (43.06) was the major practice used by the farmers followed by boundary planting and silvihorti system (Table 2) whereas all the five major agroforestry systems were recorded in Ballari district followed by four each in Yadagir, Raichur and Koppal and three systems in Kalaburagi sistrict.

Insight of the study is that the majority of the farmers preferred to grow trees on bund and on the boundary of the farm. The probable reason for inclusion of trees is being their less inhibitory impact on associated field crops in both the systems while they provided ecological and economical benefits. The availability of water was the deciding factor for farmers to integrate trees in the form of bund planting, boundary planting, silvihorti, silvipasture and block plantation. Particularly, economic benefits attracted farmers to practice silvihorti and block plantation. Silvihorti system was the major system practiced by large farmer mainly due to the better socio economic status of the farmer as well attractive and assured benefits from the system. However, variation between the districts may be due to the variation in ecological conditions and socio economic status of the farmers of the respective region. The findings are in concurrence with Behera and Dhir (2011) who in their study found that home garden was the widely accepted system and $72.6 \%$ of people were practicing it under irrigated situation in Bouda district of Odisha.
Further, they too opined that the greater adoption of home garden was because of better socioeconomic status of the farmers of the region.

\section{Species richness, density and diversity}

\section{Farm holding size}

In the irrigated ecosystem 52 tree species were recorded with species density of 9.04 $\mathrm{ha}^{-1}$ (mean number of species) and mean number of trees of $104.24 \mathrm{ha}^{-1}$ (Table 3). The higher number of tree species were recorded with medium farmer (42) followed by large farmer (41) and the lowest with small holder (39). However, differences with respect to the species density among the different categories of farmers were non-significant, whereas, significant differences occurred with number of trees per hectare. Higher number of trees per hectare was recorded with large farmer (122.48) followed by medium farmer (100.45) and small farmer (91.82) (Table 3). Shannon and Simpson diversity indices did not differ significantly, however, numerically higher Shannon diversity index was observed in the small category farmer (1.21) followed by medium farmer (1.14) and large farmer (1.09) (Table 3 and Fig. 1).

Further, the mean number of trees per hectare was significantly higher in the large farmer category. That means the land holding influenced the density of the trees on the farm. Probably, larger land holding has the flexibility to accommodate more number of trees compared to small farmer where land area becomes limiting factor to accommodate more number of trees for whom food crops/economic crops are important for livelihood. The results are in agreement with Abebe, et al., (2013) who reported increase in species richness and density with increase in the farm size. Bucagu et al., (2013) assessed the tree diversity in three categories of 
farmers of two ecological situations in Rwand and observed higher density of trees with wealthier farmer than with poor farmer. Albeit, in the study numerically higher Shannon diversity index (which takes into account of both species richness and evenness) was observed in the small farmer category. This suggests that the small farmers maintained different tree species in equal proportion on their limited farm holding to meet their multiple requirements. On the contrary large and medium farmer although had higher density of trees had lower Shannon diversity index which may be attributed to preferential selection/distribution of species or the higher concentration in terms number of individuals of any one or a few species. The results are in line with Bellow et al., (2008) who in their study on adoption of fruittree-based agroforestry systems on small farms in the subtropical highlands concluded that the maintenance of diversified trees within a small farm area is an element of their livelihood strategy.

\section{Agroforestry systems}

Differences in the mean number of species per hectare among the agroforestry systems revealed non-significant variations (Table 3). However, significantly higher number of trees per hectare was found in silvihorti system (123) followed by boundary planting (105.43) and bund planting (93.10) whereas least number of trees per hectare were recorded in silvipasture system (48.00) followed by scattered planting (69.33). Interestingly, significantly higher Shannon diversity index was observed in scattered planting (1.79) followed by silvipasture system (1.72) whereas lower value was recorded in bund planting (1.04) (Table 3).

The higher density of trees was recorded in silvihorti system and boundary planting which may be attributed to the fact that farmers plant thickly on the border of the farm or orchard mainly for the purposes such as getting protection as live fence from stray cattle menace, desiccating wind, sun scorching, for soil conservation, land demarcation etc. besides getting additional income if any. The results are in concurrence with Abebe, et al., (2013) who also reported increase in species richness and density with increase in the farm size.

Interestingly, significantly higher Shannon diversity index was observed in the scattered planting. This suggests that different tree species are proportionally distributed in the scattered planting. The lower Shannon index in bund planting and boundary planting indicates that non proportional distribution of tree species or the higher concentration in terms number of individuals of any one or a few species. The results are in conformity with Henry et al., (2009) who also reported higher diversity in homestead and farm land where different trees distributed were mostly in equal proportion. They also observed lower diversity in wood lot and hedge planting.

\section{Different districts}

The study found significantly higher mean number of species per hectare in Kalaburagi district (11.25) followed by Bidar (9.08) and lower mean number of species per hectare was recorded in Koppal (7.75) followed by Ballari (8.58) (Table 3 and Fig. 2). However, mean number of trees and diversity indices varied non- significantly among the districts. The higher species density in Kalaburagi and Bidar may be attributed to substantially higher rainfall in these districts which favoured the growth of the tree species, and the other factor is major agroforestry systems practiced by the farmers in this region were boundary planting, bund planting and scattered planting which contributed for the species diversity in the respective districts. 
Table.1 Agroforestry systems followed by different categories of farmers in north-eastern parts of Karnataka under irrigated ecosystem

\begin{tabular}{|c|c|c|c|c|}
\hline \multirow{3}{*}{ Agroforestry systems } & \multirow{2}{*}{\multicolumn{3}{|c|}{$\begin{array}{c}\text { Farmers following the agroforestry systems (\%) } \\
\text { Categories of Farmers }\end{array}$}} & \multirow{3}{*}{$\begin{array}{c}\text { Average } \\
(\mathrm{N}=72)\end{array}$} \\
\hline & & & & \\
\hline & Large $(n=21)$ & Medium (n=29) & Small $(n=22)$ & \\
\hline Bund planting & 28.57 & 55.17 & 40.91 & $43.06( \pm 0.70)$ \\
\hline Boundary planting & 14.29 & 17.24 & 27.27 & $19.44( \pm 0.80)$ \\
\hline Scattered planting & 4.76 & 3.45 & 4.55 & $4.17( \pm 1.00)$ \\
\hline Silvihorti system & 14.29 & 10.34 & 13.64 & $12.50( \pm 0.87)$ \\
\hline Silvipasture system & 0.00 & 3.45 & 4.55 & $2.78( \pm 0.71)$ \\
\hline Bund + block plantation & 4.76 & 6.90 & 4.55 & $5.56( \pm 0.82)$ \\
\hline Boundary + block plantation & 4.76 & 0.00 & 4.55 & $2.78( \pm 1.41)$ \\
\hline Silvihorti +block plantation & 28.57 & 0.00 & 0.00 & 8.33 \\
\hline Bund + boundary planting & 0.00 & 3.45 & 0.00 & 1.39 \\
\hline
\end{tabular}

Note: Values in the parentheses indicate the standard deviation

Table.2 Agroforestry systems followed by farmers in different districts of north-eastern parts of Karnataka under irrigated ecosystem

\begin{tabular}{|c|c|c|c|c|c|c|c|c|c|c|}
\hline \multirow{2}{*}{$\begin{array}{c}\text { Districts/Agroforestry } \\
\text { systems }\end{array}$} & \multicolumn{10}{|c|}{ Farmers following the agroforestry systems $(\%)$} \\
\hline & BND & BDR & SCP & SH & SP & $\mathrm{BND}+\mathrm{BP}$ & BDR+BP & $\mathbf{S H}+\mathbf{B P}$ & BND+BDR & SD \\
\hline $\operatorname{Bidar}(n=12)$ & 75.00 & 8.33 & 0.00 & 0.00 & 0.00 & 0.00 & 8.33 & 8.33 & 0.00 & 2.90 \\
\hline Kalaburagi $(n=12)$ & 33.33 & 41.67 & 0.00 & 16.67 & 0.00 & 8.33 & 0.00 & 0.00 & 0.00 & 1.78 \\
\hline Yadgir $(n=12)$ & 41.67 & 8.33 & 8.33 & 16.67 & 0.00 & 8.33 & 0.00 & 8.33 & 8.33 & 3.47 \\
\hline Raichur $(n=12)$ & 41.67 & 25.00 & 8.33 & 8.33 & 0.00 & 8.33 & 8.33 & 0.00 & 0.00 & 2.42 \\
\hline Ballari $(n=12)$ & 33.33 & 16.67 & 8.33 & 25.00 & 8.33 & 8.33 & 0.00 & 0.00 & 0.00 & 1.93 \\
\hline Koppal $(n=12)$ & 33.33 & 16.67 & 0.00 & 8.33 & 8.33 & 0.00 & 0.00 & 33.33 & 0.00 & 3.47 \\
\hline Average $(n=72)$ & 43.06 & 19.44 & 4.17 & 12.50 & 2.78 & 5.56 & 2.78 & 8.33 & 1.39 & 2.66 \\
\hline
\end{tabular}

BP -Block plantation, SD-Standard deviation. 
Table.3 Species richness, mean species density and mean number of trees per hectare in different land holding size, agroforestry systems and districts under irrigated ecosystem in north- eastern parts of Karnataka

\begin{tabular}{|c|c|c|c|c|c|}
\hline & $\begin{array}{l}\text { Species richness } \\
\text { (Total number) }\end{array}$ & $\begin{array}{c}\text { Species density } \\
\left(\text { ha }^{-1}\right)\end{array}$ & $\begin{array}{c}\text { Tree density } \\
\quad\left(\mathrm{ha}^{-1}\right)\end{array}$ & $\mathbf{H}$ & $\lambda$ \\
\hline \multicolumn{6}{|l|}{ Categories of farmer (a) } \\
\hline Large $(n=21)$ & 41 & $9.67( \pm 2.50)$ & $122.48^{\mathrm{b}}( \pm 40.23)$ & $1.09( \pm 0.42)$ & $0.53( \pm 0.19)$ \\
\hline Medium $(n=29)$ & 42 & $8.48( \pm 2.70)$ & $100.45^{\mathrm{a}}( \pm 38.06)$ & $1.14( \pm 0.43)$ & $0.48( \pm 0.20)$ \\
\hline Small $(n=22)$ & 39 & $9.18( \pm 2.94)$ & $91.82^{\mathrm{a}}( \pm 29.82)$ & $1.21( \pm 0.40)$ & $0.47( \pm 0.15)$ \\
\hline F test $(\mathbf{P})$ & & $0.308^{\mathrm{NS}}$ & $<0.05$ & $0.646^{\mathrm{NS}}$ & $0.528^{\mathrm{NS}}$ \\
\hline \multicolumn{6}{|l|}{ Agroforestry systems (b) } \\
\hline Bund planting $(\mathrm{n}=31)$ & 37 & $8.45( \pm 2.87)$ & $93.10^{\mathrm{abc}}( \pm 28.64)$ & $1.04^{\mathrm{a}}( \pm 0.31)$ & $0.53^{\mathrm{c}}( \pm 0.14)$ \\
\hline Boundary planting $(\mathrm{n}=14)$ & 30 & $9.00( \pm 2.96)$ & $105.43^{\mathrm{bc}}( \pm 33.13)$ & $1.17^{\mathrm{ab}}( \pm 0.52)$ & $0.49^{\mathrm{bc}}( \pm 0.22)$ \\
\hline Scattered planting $(n=3)$ & 21 & $10.00( \pm 2.65)$ & $69.33^{\mathrm{ab}}( \pm 4.73)$ & $1.79^{c}( \pm 0.43)$ & $0.23^{\mathrm{ab}}( \pm 0.10)$ \\
\hline Silvihorti system $(\mathrm{n}=9)$ & 35 & $9.89( \pm 2.26)$ & $123.00^{\mathrm{cd}}( \pm 30.82)$ & $1.21^{\mathrm{abc}}( \pm 0.37)$ & $0.46^{\mathrm{abc}}( \pm 0.18)$ \\
\hline Silvipasture system $(\mathrm{n}=2)$ & 15 & $8.00( \pm 0.00)$ & $48.00^{\mathrm{a}}( \pm 5.66)$ & $1.72^{\mathrm{bc}}( \pm 0.08)$ & $0.22^{\mathrm{a}}( \pm 0.02)$ \\
\hline Bund + block plantation $(n=4)$ & 24 & $9.25( \pm 2.75)$ & $99.75^{\mathrm{bc}}( \pm 43.25)$ & $1.01^{\mathrm{a}}( \pm 0.46)$ & $0.56^{\mathrm{c}}( \pm 0.22)$ \\
\hline $\begin{array}{l}\text { Boundary + block plantation } \\
(\mathrm{n}=2)\end{array}$ & 17 & $11.50( \pm 0.71)$ & $117.50^{\mathrm{bcd}}( \pm 10.61)$ & $1.45^{\mathrm{abc}}( \pm 0.01)$ & $0.38^{\mathrm{abc}}( \pm 0.01)$ \\
\hline $\begin{array}{l}\text { Silvihorti +block plantation } \\
(\mathrm{n}=6)\end{array}$ & 29 & $9.67( \pm 3.14)$ & $154.50^{\mathrm{d}}( \pm 51.09)$ & $1.10^{\mathrm{a}}( \pm 0.43)$ & $0.51^{\mathrm{c}}( \pm 0.21)$ \\
\hline $\begin{array}{l}\text { Bund + boundary planting } \\
(\mathrm{n}=1)\end{array}$ & 10 & 10.00 & 171.00 & 0.62 & 0.77 \\
\hline F test $(\mathbf{P})$ & & $0.691^{\mathrm{NS}}$ & $<0.05$ & $<0.05$ & 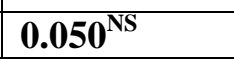 \\
\hline \multicolumn{6}{|l|}{ Districts (c) } \\
\hline Bidar $(n=12)$ & 26 & $9.08^{\mathrm{a}}( \pm 2.78)$ & $93.75^{\mathrm{a}}( \pm 19.64)$ & $1.04( \pm 0.41)$ & $0.56( \pm 0.17)$ \\
\hline Kalaburagi $(n=12)$ & 32 & $11.25^{\mathrm{b}}( \pm 1.91)$ & $103.58^{\mathrm{ab}}( \pm 14.43)$ & $1.23( \pm 0.30)$ & $0.49( \pm 0.13)$ \\
\hline Yadgir $(n=12)$ & 29 & $8.67^{\mathrm{a}}( \pm 2.90)$ & $109.75^{\mathrm{ab}}( \pm 39.43)$ & $0.93( \pm 0.41)$ & $0.59( \pm 0.20)$ \\
\hline Raichur $(\mathrm{n}=12)$ & 30 & $8.92^{\mathrm{a}}( \pm 2.07)$ & $85.67^{\mathrm{a}}( \pm 25.27)$ & $1.26( \pm 0.31)$ & $0.43( \pm 0.23)$ \\
\hline Bellary $(n=12)$ & 33 & $8.58^{\mathrm{a}}( \pm 3.09)$ & $105.33^{\mathrm{ab}}( \pm 45.48)$ & $1.24( \pm 0.58)$ & $0.44( \pm 0.17)$ \\
\hline Koppal $(n=12)$ & 33 & $7.75^{\mathrm{a}}( \pm 2.60)$ & $127.33^{\mathrm{b}}( \pm 57.79)$ & $1.17( \pm 0.39)$ & $0.44( \pm 0.18)$ \\
\hline F test $(\mathbf{P})$ & & $<0.05$ & $\mathbf{0 . 1 2 6}^{\mathrm{NS}}$ & $\mathbf{0 . 3 0 0}^{\mathrm{NS}}$ & $0.110^{\mathrm{NS}}$ \\
\hline Average $(\mathrm{N}=72)^{*}$ & 52 & $9.04( \pm 2.72)$ & $\mathbf{1 0 4 . 2 4}( \pm 37.98)$ & $1.15( \pm 0.41)$ & $0.49( \pm 0.18)$ \\
\hline
\end{tabular}

Note: H-Shannon’s Index, $\lambda$ - Simpson Index, Values in the parentheses indicates the standard deviation, ${ }^{\mathrm{a}, \mathrm{b}}$ Values within the column with same letter are not significantly different at $\mathrm{P}<0.05$ 
Table.4 Dominant tree species found in different land holding size of north-eastern parts of Karnataka under irrigated ecosystem

\begin{tabular}{|c|c|c|c|c|c|c|c|c|c|c|c|c|c|}
\hline \multirow{2}{*}{$\begin{array}{l}\text { SI. } \\
\text { No. }\end{array}$} & \multirow[t]{2}{*}{ Scientific name } & \multicolumn{3}{|c|}{ Large farmer $(n=21)$} & \multicolumn{3}{|c|}{ Medium farmer (n=29) } & \multicolumn{3}{|c|}{ Small farmer $(n=22)$} & \multicolumn{3}{|c|}{ Grand total } \\
\hline & & $\begin{array}{c}\text { Total } \\
\text { No. }\end{array}$ & RD & $\mathbf{R F}$ & $\begin{array}{c}\text { Total } \\
\text { No. }\end{array}$ & RD & $\mathbf{R F}$ & $\begin{array}{c}\text { Total } \\
\text { No. }\end{array}$ & RD & $\mathbf{R F}$ & $\begin{array}{c}\text { Total } \\
\text { No. }\end{array}$ & RD & RF \\
\hline 1 & Tectona grandis & 1696 & 65.94 & 95.24 & 1782 & 61.17 & 93.10 & 1249 & 61.83 & 95.45 & 4817 & 64.01 & 94.44 \\
\hline 2 & Azadirachta indica & 258 & 10.03 & 100.00 & 238 & 8.17 & 100.00 & 234 & 11.58 & 100.00 & 660 & 8.77 & 100.00 \\
\hline 3 & Cocos nucifera & 106 & 4.12 & 47.62 & 376 & 12.91 & 62.07 & 95 & 4.70 & 54.55 & 577 & 7.67 & 55.56 \\
\hline 4 & Grevillea robusta & 105 & 4.08 & 9.52 & 78 & 2.68 & 6.90 & 94 & 4.65 & 9.09 & 277 & 3.68 & 8.33 \\
\hline 5 & Melia dubia & 55 & 2.14 & 9.52 & 45 & 1.54 & 6.90 & 20 & 0.99 & 4.55 & 120 & 1.59 & 6.94 \\
\hline 6 & Tamarindus indica & 52 & 2.02 & 71.43 & 23 & 0.79 & 37.93 & 32 & 1.58 & 63.64 & 107 & 1.42 & 55.56 \\
\hline 7 & Acacia nilotica & 34 & 1.32 & 47.62 & 29 & 1.00 & 31.03 & 42 & 2.08 & 59.09 & 105 & 1.40 & 44.44 \\
\hline 8 & Mangifera indica & 31 & 1.21 & 61.90 & 35 & 1.20 & 41.38 & 20 & 0.99 & 54.55 & 86 & 1.14 & 51.39 \\
\hline 9 & Leucaena leucocephala & 27 & 1.05 & 42.86 & 75 & 2.57 & 58.62 & 16 & 0.79 & 36.36 & 118 & 1.57 & 47.22 \\
\hline 10 & Eucalyptus tereticornis & 23 & 0.89 & 14.29 & 27 & 0.93 & 17.24 & 23 & 1.14 & 18.18 & 73 & 0.97 & 16.67 \\
\hline 11 & Syzygium cumini & 21 & 0.82 & 42.86 & 18 & 0.62 & 27.59 & 24 & 1.19 & 45.45 & 63 & 0.84 & 37.50 \\
\hline 12 & Annona squamosa & 20 & 0.78 & 38.10 & 26 & 0.89 & 34.48 & 29 & 1.44 & 40.91 & 75 & 1.00 & 37.50 \\
\hline 13 & Psidium guajava & 16 & 0.62 & 38.10 & 12 & 0.41 & 24.14 & 13 & 0.64 & 27.27 & 41 & 0.54 & 29.17 \\
\hline 14 & Gliricidia sepium & 15 & 0.58 & 4.76 & 5 & 0.17 & 3.45 & $\mathbf{0}$ & 0.00 & 0.00 & 20 & 0.27 & 2.78 \\
\hline 15 & Murraya koenigii & 13 & 0.51 & 23.81 & 6 & 0.21 & 13.79 & 13 & 0.64 & 27.27 & 32 & 0.43 & 20.83 \\
\hline 16 & Ziziphus mauritiana & 11 & 0.43 & 38.10 & 18 & 0.62 & 37.93 & 12 & 0.59 & 45.45 & 41 & 0.54 & 40.28 \\
\hline 17 & Citrus limon & 11 & 0.43 & 33.33 & 14 & 0.48 & 24.14 & 19 & 0.94 & 31.82 & 44 & 0.58 & 29.17 \\
\hline 18 & Melia azedarachta & 11 & 0.43 & 14.29 & 5 & 0.17 & 6.90 & 11 & 0.54 & 9.09 & 27 & 0.36 & 9.72 \\
\hline 19 & Santalum album & 8 & 0.31 & 23.81 & 13 & 0.45 & 27.59 & 6 & 0.30 & 22.73 & 27 & 0.36 & 25.00 \\
\hline 20 & Phyllanthus emblica & 8 & 0.31 & 23.81 & 2 & 0.07 & 6.90 & 1 & 0.05 & 4.55 & 11 & 0.15 & 11.11 \\
\hline 21 & Balanites roxburghii & 7 & 0.27 & 9.52 & 4 & 0.14 & 6.90 & 5 & 0.25 & 9.09 & 16 & 0.21 & 8.33 \\
\hline 22 & Prosopis cineraria & 5 & 0.19 & 19.05 & 5 & 0.17 & 17.24 & 9 & 0.45 & 22.73 & 19 & 0.25 & 19.44 \\
\hline 23 & Terminalia catappa & 5 & 0.19 & 19.05 & 2 & 0.07 & 6.90 & 5 & 0.25 & 9.09 & 12 & 0.16 & 11.11 \\
\hline 24 & Albizia lebbek & 4 & 0.16 & 14.29 & 3 & 0.10 & 10.34 & 1 & 0.05 & 4.55 & 8 & 0.11 & 9.72 \\
\hline 25 & Achras sapota & 3 & 0.12 & 9.52 & 11 & 0.38 & 13.79 & 8 & 0.40 & 18.18 & 22 & 0.29 & 13.89 \\
\hline 26 & Hardwickia binata & 3 & 0.12 & 9.52 & 4 & 0.14 & 10.34 & 3 & 0.15 & 9.09 & 10 & 0.13 & 9.72 \\
\hline 27 & Bambusa arundinacea & 3 & 0.12 & 9.52 & 8 & 0.27 & 17.24 & $\mathbf{1}$ & 0.05 & 4.55 & 12 & 0.16 & 11.11 \\
\hline 28 & Ficus glomerata & 3 & 0.12 & 9.52 & 2 & 0.07 & 6.90 & $\mathbf{1}$ & 0.05 & 4.55 & 6 & 0.08 & 6.94 \\
\hline 29 & Pithecellobium dulce & 3 & 0.12 & 14.29 & 3 & 0.10 & 6.90 & 2 & 0.10 & 4.55 & 8 & 0.11 & 8.33 \\
\hline 30 & Cassia fistula & 2 & 0.08 & 9.52 & 3 & 0.10 & 6.90 & $\mathbf{0}$ & 0.00 & 0.00 & 5 & 0.07 & 5.56 \\
\hline 31 & Moringa oleifera & 2 & 0.08 & 9.52 & 12 & 0.41 & 20.69 & 6 & 0.30 & 18.18 & 20 & 0.27 & 16.67 \\
\hline 32 & Ailanthus excelsa & 2 & 0.08 & 9.52 & 1 & 0.03 & 3.45 & $\mathbf{0}$ & 0.00 & 0.00 & 3 & 0.04 & 4.17 \\
\hline
\end{tabular}




\begin{tabular}{|c|c|c|c|c|c|c|c|c|c|c|c|c|c|}
\hline \multirow{2}{*}{$\begin{array}{l}\text { Sl. } \\
\text { No. }\end{array}$} & \multirow[t]{2}{*}{ Scientific name } & \multicolumn{3}{|c|}{ Large farmer $(n=21)$} & \multicolumn{3}{|c|}{ Medium farmer $(n=29)$} & \multicolumn{3}{|c|}{ Small farmer $(n=22)$} & \multicolumn{3}{|c|}{ Grand total } \\
\hline & & $\begin{array}{l}\text { Total } \\
\text { No. }\end{array}$ & RD & $\mathbf{R F}$ & $\begin{array}{l}\text { Total } \\
\text { No. }\end{array}$ & RD & $\mathbf{R F}$ & $\begin{array}{c}\text { Total } \\
\text { No. }\end{array}$ & RD & $\mathbf{R F}$ & $\begin{array}{l}\text { Total } \\
\text { No. }\end{array}$ & RD & $\mathbf{R F}$ \\
\hline 33 & Aegle marmelos & 1 & 0.04 & 4.76 & 3 & 0.10 & 6.90 & 1 & 0.05 & 4.55 & 5 & 0.03 & 5.56 \\
\hline 34 & Butea monosperma & 1 & 0.04 & 4.76 & 1 & 0.03 & 3.45 & $\mathbf{0}$ & 0.00 & 0.00 & 2 & 0.15 & 2.78 \\
\hline 35 & Morinda pubescens & 1 & 0.04 & 4.76 & 9 & 0.31 & 13.79 & 1 & 0.05 & 4.55 & 11 & 0.01 & 8.33 \\
\hline 36 & Pongamia pinnata & 1 & 0.04 & 4.76 & $\mathbf{0}$ & 0.00 & 0.00 & 4 & 0.20 & 9.09 & 5 & 0.01 & 4.17 \\
\hline 37 & Borassus flabellifer & 1 & 0.04 & 4.76 & $\mathbf{0}$ & 0.00 & 0.00 & $\mathbf{0}$ & 0.00 & 0.00 & 1 & 0.05 & 1.39 \\
\hline 38 & Acacia ferruginea & 1 & 0.04 & 4.76 & $\mathbf{0}$ & 0.00 & 0.00 & $\mathbf{0}$ & 0.00 & 0.00 & 1 & 0.03 & 1.39 \\
\hline 39 & Acacia auriculiformis & 1 & 0.04 & 4.76 & $\mathbf{0}$ & 0.00 & 0.00 & $\mathbf{0}$ & 0.00 & 0.00 & 1 & 0.04 & 1.39 \\
\hline 40 & Wrightia tinctoria & 1 & 0.04 & 4.76 & 3 & 0.10 & 6.90 & $\mathbf{0}$ & 0.00 & 0.00 & 4 & 0.07 & 4.17 \\
\hline 41 & Michelia champaca & 1 & 0.04 & 4.76 & 1 & 0.03 & 3.45 & $\mathbf{0}$ & 0.00 & 0.00 & 2 & 0.07 & 2.78 \\
\hline 42 & Albizia procera & $\mathbf{0}$ & 0.00 & 0.00 & 1 & 0.03 & 3.45 & 2 & 0.10 & 9.09 & 3 & 0.04 & 4.17 \\
\hline 43 & $\begin{array}{l}\text { Casuarina } \\
\text { equisetifolia }\end{array}$ & $\mathbf{0}$ & 0.00 & 0.00 & $\mathbf{0}$ & 0.00 & 0.00 & 5 & 0.25 & 4.55 & 5 & 0.03 & 1.39 \\
\hline 44 & Sesbania grandiflora & $\mathbf{0}$ & 0.00 & 0.00 & $\mathbf{0}$ & 0.00 & 0.00 & 5 & 0.25 & 9.09 & 5 & 0.07 & 2.78 \\
\hline 45 & Madhuca indica & $\mathbf{0}$ & 0.00 & 0.00 & $\mathbf{0}$ & 0.00 & 0.00 & 3 & 0.15 & 9.09 & 3 & 0.04 & 2.78 \\
\hline 46 & Ficus religiosa & $\mathbf{0}$ & 0.00 & 0.00 & $\mathbf{0}$ & 0.00 & 0.00 & 2 & 0.10 & 4.55 & 2 & 0.03 & 1.39 \\
\hline 47 & Chloroxylon swietenia & $\mathbf{0}$ & 0.00 & 0.00 & 5 & 0.17 & 6.90 & $\mathbf{0}$ & 0.00 & 0.00 & 5 & 0.07 & 2.78 \\
\hline 48 & Phoenixa dactylifera & $\mathbf{0}$ & 0.00 & 0.00 & 3 & 0.10 & 6.90 & $\mathbf{0}$ & 0.00 & 0.00 & 3 & 0.04 & 2.78 \\
\hline 49 & Limonia acidissima & $\mathbf{0}$ & 0.00 & 0.00 & $\mathbf{0}$ & 0.00 & 0.00 & 1 & 0.05 & 4.55 & 1 & 0.01 & 1.39 \\
\hline 50 & Delonix regia. & $\mathbf{0}$ & 0.00 & 0.00 & $\mathbf{0}$ & 0.00 & 0.00 & 2 & 0.10 & 4.55 & 2 & 0.03 & 1.39 \\
\hline 51 & Muntingia calubura & $\mathbf{0}$ & 0.00 & 0.00 & 1 & 0.03 & 3.45 & $\mathbf{0}$ & 0.00 & 0.00 & 1 & 0.01 & 1.39 \\
\hline 52 & Ficus lacor & $\mathbf{0}$ & 0.00 & 0.00 & 1 & 0.03 & 3.45 & $\mathbf{0}$ & 0.00 & 0.00 & 1 & 0.01 & 1.39 \\
\hline & $\begin{array}{l}\text { Total number of } \\
\text { trees }\end{array}$ & 2572 & & & 2913 & & & 2020 & & & 7525 & & \\
\hline & $\begin{array}{l}\text { Mean number of } \\
\text { trees ha }^{-1}\end{array}$ & 122.48 & & & 100.45 & & & 91.82 & & & 104.51 & & \\
\hline
\end{tabular}

Note: RD- Relative Density, RF-Relative frequency 
Table.5 Dominant tree species found in different agroforestry systems of north-eastern parts of Karnataka under irrigated ecosystem

\begin{tabular}{|c|c|c|c|c|c|c|c|c|c|c|c|c|c|c|c|c|c|c|c|}
\hline \multirow[t]{2}{*}{$\begin{array}{l}\text { Sl. } \\
\text { No. }\end{array}$} & \multirow[t]{2}{*}{ Scientific name } & \multicolumn{2}{|c|}{ BND(n=31) } & \multicolumn{2}{|c|}{ BDR(n=14) } & \multicolumn{2}{|c|}{$\operatorname{SCP}(n=3)$} & \multicolumn{2}{|c|}{ SH(n=9) } & \multicolumn{2}{|c|}{$\mathbf{S P}(n=2)$} & \multicolumn{2}{|c|}{$\mathrm{BND}+\mathrm{BP}(\mathrm{n}=4)$} & \multicolumn{2}{|c|}{$\mathrm{BDR}+\mathrm{BP}(\mathrm{n}=2)$} & \multicolumn{2}{|c|}{$\begin{array}{c}\mathrm{SH}+\mathrm{BP} \\
(\mathbf{n}=\mathbf{6})\end{array}$} & \multicolumn{2}{|c|}{$\begin{array}{c}\text { BND+BDR } \\
(n=1)\end{array}$} \\
\hline & & $\begin{array}{c}\text { Total } \\
\text { No. }\end{array}$ & RD & $\begin{array}{c}\text { Total } \\
\text { No. }\end{array}$ & RD & $\begin{array}{c}\text { Total } \\
\text { No. }\end{array}$ & RD & $\begin{array}{c}\text { Total } \\
\text { No. }\end{array}$ & RD & $\begin{array}{c}\text { Total } \\
\text { No. }\end{array}$ & RD & $\begin{array}{c}\text { Total } \\
\text { No. }\end{array}$ & RD & $\begin{array}{c}\text { Total } \\
\text { No. }\end{array}$ & RD & $\begin{array}{c}\text { Total } \\
\text { No. }\end{array}$ & RD & $\begin{array}{c}\text { Total } \\
\text { No. }\end{array}$ & RD \\
\hline 1 & Tectona grandis & 1951 & 67.60 & 922 & 62.47 & 25 & 12.02 & 661 & 59.71 & 9 & 9.38 & 307 & 76.94 & 137 & 58.30 & 565 & 60.95 & 150 & 87.72 \\
\hline 2 & Azadirachta indica & 291 & 10.08 & 141 & 9.55 & 45 & 21.63 & 75 & 6.78 & 22 & 22.92 & 32 & 8.02 & 17 & 7.23 & 102 & 11.00 & 5 & 2.92 \\
\hline 3 & Cocos nucifera & 233 & 8.07 & 121 & 8.20 & 51 & 24.52 & 127 & 11.47 & 15 & 15.63 & 3 & 0.75 & 18 & 7.66 & 7 & 0.76 & 2 & 1.17 \\
\hline 4 & Tamarindus indica & 54 & 1.87 & 18 & 1.22 & 6 & 2.88 & 14 & 1.26 & 3 & 3.13 & 3 & 0.75 & $\mathbf{0}$ & 0.00 & 9 & 0.97 & $\mathbf{0}$ & 0.00 \\
\hline 5 & Leucaena leисосерhala & 45 & 1.56 & 26 & 1.76 & 6 & 2.88 & 9 & 0.81 & 4 & 4.17 & 17 & 4.26 & $\mathbf{1}$ & 0.43 & 6 & 0.65 & 4 & 2.34 \\
\hline 6 & Acacia nilotica & 41 & 1.42 & 30 & 2.03 & 3 & 1.44 & 10 & 0.90 & $\mathbf{0}$ & 0.00 & 4 & 1.00 & 5 & 2.13 & 10 & 1.08 & 2 & 1.17 \\
\hline 7 & Mangifera indica & 38 & 1.32 & 13 & 0.88 & 8 & 3.85 & 7 & 0.63 & 2 & 2.08 & 2 & 0.50 & 1 & 0.43 & 13 & 1.40 & 2 & 1.17 \\
\hline 8 & Annona squamosa & 27 & 0.94 & 32 & 2.17 & 4 & 1.92 & 10 & 0.90 & $\mathbf{0}$ & 0.00 & 2 & 0.50 & $\mathbf{0}$ & 0.00 & $\mathbf{0}$ & 0.00 & $\mathbf{0}$ & 0.00 \\
\hline 9 & Eucalyptus tereticornis & 27 & 0.94 & 16 & 1.08 & $\mathbf{0}$ & 0.00 & 2 & 0.18 & $\mathbf{0}$ & 0.00 & $\mathbf{0}$ & 0.00 & 23 & 9.79 & 5 & 0.54 & $\mathbf{0}$ & 0.00 \\
\hline 10 & Psidium guajava & 21 & 0.73 & 11 & 0.75 & $\mathbf{0}$ & 0.00 & 4 & 0.36 & $\mathbf{0}$ & 0.00 & 2 & 0.50 & $\mathbf{0}$ & 0.00 & 3 & 0.32 & $\mathbf{0}$ & 0.00 \\
\hline 11 & Syzygium cumini & 17 & 0.59 & 3 & 0.20 & 4 & 1.92 & 18 & 1.63 & 4 & 4.17 & 1 & 0.25 & 2 & 0.85 & 14 & 1.51 & $\mathbf{0}$ & 0.00 \\
\hline 12 & Murraya koenigii & 17 & 0.59 & $\mathbf{0}$ & 0.00 & $\mathbf{0}$ & 0.00 & 1 & 0.09 & 4 & 4.17 & 3 & 0.75 & 2 & 0.85 & 5 & 0.54 & $\mathbf{0}$ & 0.00 \\
\hline 13 & Ziziphus mauritiana & 16 & 0.55 & 11 & 0.75 & 1 & 0.48 & 2 & 0.18 & 5 & 5.21 & 1 & 0.25 & 2 & 0.85 & 2 & 0.22 & 1 & 0.58 \\
\hline 14 & Melia azedarachta & 16 & 0.55 & $\mathbf{0}$ & 0.00 & 10 & 4.81 & $\mathbf{0}$ & 0.00 & $\mathbf{0}$ & 0.00 & 1 & 0.25 & $\mathbf{0}$ & 0.00 & $\mathbf{0}$ & 0.00 & $\mathbf{0}$ & 0.00 \\
\hline 15 & Citrus limon & 13 & 0.45 & 3 & 0.20 & 5 & 2.40 & 6 & 0.54 & 4 & 4.17 & 7 & 1.75 & 3 & 1.28 & 3 & 0.32 & $\mathbf{0}$ & 0.00 \\
\hline 16 & Santalum album & 10 & 0.35 & 8 & 0.54 & 2 & 0.96 & 3 & 0.27 & $\mathbf{0}$ & 0.00 & $\mathbf{0}$ & 0.00 & $\mathbf{0}$ & 0.00 & 3 & 0.32 & 1 & 0.58 \\
\hline 17 & Balanites roxburghii & 9 & 0.31 & $\mathbf{0}$ & 0.00 & 4 & 1.92 & 0 & 0.00 & $\mathbf{0}$ & 0.00 & $\mathbf{0}$ & 0.00 & $\mathbf{0}$ & 0.00 & 3 & 0.32 & 0 & 0.00 \\
\hline 18 & Achras sapota & 8 & 0.28 & 7 & 0.47 & $\mathbf{0}$ & 0.00 & 2 & 0.18 & $\mathbf{0}$ & 0.00 & 1 & 0.25 & 2 & 0.85 & 2 & 0.22 & $\mathbf{0}$ & 0.00 \\
\hline 19 & Grevillea robusta & 8 & 0.28 & 75 & 5.08 & $\mathbf{0}$ & 0.00 & 70 & 6.32 & 19 & 19.79 & $\mathbf{0}$ & 0.00 & $\mathbf{0}$ & 0.00 & 105 & 11.33 & $\mathbf{0}$ & 0.00 \\
\hline 20 & Hardwickia binata & 7 & 0.24 & 3 & 0.20 & $\mathbf{0}$ & 0.00 & $\mathbf{0}$ & 0.00 & $\mathbf{0}$ & 0.00 & $\mathbf{0}$ & 0.00 & $\mathbf{0}$ & 0.00 & $\mathbf{0}$ & 0.00 & $\mathbf{0}$ & 0.00 \\
\hline 21 & Prosopis cineraria & 5 & 0.17 & 6 & 0.41 & $\mathbf{0}$ & 0.00 & 5 & 0.45 & $\mathbf{0}$ & 0.00 & 3 & 0.75 & $\mathbf{0}$ & 0.00 & $\mathbf{0}$ & 0.00 & $\mathbf{0}$ & 0.00 \\
\hline 22 & Morinda pubescens & 5 & 0.17 & 5 & 0.34 & $\mathbf{0}$ & 0.00 & 1 & 0.09 & $\mathbf{0}$ & 0.00 & $\mathbf{0}$ & 0.00 & $\mathbf{0}$ & 0.00 & $\mathbf{0}$ & 0.00 & $\mathbf{0}$ & 0.00 \\
\hline 23 & Bambusa arundinacea & 4 & 0.14 & 2 & 0.14 & $\mathbf{0}$ & 0.00 & 2 & 0.18 & $\mathbf{0}$ & 0.00 & $\mathbf{1}$ & 0.25 & 2 & 0.85 & $\mathbf{1}$ & 0.11 & $\mathbf{0}$ & 0.00 \\
\hline 24 & Cassia fistula & 3 & 0.10 & $\mathbf{0}$ & 0.00 & 1 & 0.48 & $\mathbf{0}$ & 0.00 & $\mathbf{0}$ & 0.00 & $\mathbf{0}$ & 0.00 & $\mathbf{0}$ & 0.00 & 1 & 0.11 & $\mathbf{0}$ & 0.00 \\
\hline 25 & Wrightia tinctoria & 3 & 0.10 & 1 & 0.07 & $\mathbf{0}$ & 0.00 & $\mathbf{0}$ & 0.00 & $\mathbf{0}$ & 0.00 & $\mathbf{0}$ & 0.00 & $\mathbf{0}$ & 0.00 & $\mathbf{0}$ & 0.00 & $\mathbf{0}$ & 0.00 \\
\hline 26 & Albizia lebbek & 2 & 0.07 & 1 & 0.07 & $\mathbf{0}$ & 0.00 & 1 & 0.09 & $\mathbf{0}$ & 0.00 & 1 & 0.25 & $\mathbf{0}$ & 0.00 & 3 & 0.32 & $\mathbf{0}$ & 0.00 \\
\hline 27 & Terminalia catappa & 2 & 0.07 & $\mathbf{0}$ & 0.00 & $\mathbf{0}$ & 0.00 & 8 & 0.72 & 1 & 1.04 & $\mathbf{0}$ & 0.00 & $\mathbf{0}$ & 0.00 & 1 & 0.11 & 0 & 0.00 \\
\hline 28 & Ficus religiosa & 2 & 0.07 & $\mathbf{0}$ & 0.00 & $\mathbf{0}$ & 0.00 & $\mathbf{0}$ & 0.00 & $\mathbf{0}$ & 0.00 & $\mathbf{0}$ & 0.00 & $\mathbf{0}$ & 0.00 & $\mathbf{0}$ & 0.00 & $\mathbf{0}$ & 0.00 \\
\hline 29 & Chloroxylon swietenia & 2 & 0.07 & $\mathbf{0}$ & 0.00 & 3 & 1.44 & $\mathbf{0}$ & 0.00 & $\mathbf{0}$ & 0.00 & $\mathbf{0}$ & 0.00 & $\mathbf{0}$ & 0.00 & $\mathbf{0}$ & 0.00 & $\mathbf{0}$ & 0.00 \\
\hline 30 & Phoenixa dactylifera & 2 & 0.07 & 1 & 0.07 & $\mathbf{0}$ & 0.00 & $\mathbf{0}$ & 0.00 & $\mathbf{0}$ & 0.00 & $\mathbf{0}$ & 0.00 & $\mathbf{0}$ & 0.00 & $\mathbf{0}$ & 0.00 & $\mathbf{0}$ & 0.00 \\
\hline 31 & Phyllanthus emblica & 1 & 0.03 & $\mathbf{0}$ & 0.00 & $\mathbf{0}$ & 0.00 & 6 & 0.54 & $\mathbf{0}$ & 0.00 & 1 & 0.25 & $\mathbf{0}$ & 0.00 & 3 & 0.32 & $\mathbf{0}$ & 0.00 \\
\hline
\end{tabular}

Note: BND-Bund planting, BDR-Boundary planting, SCP, Scatterd planting SH-Silvihorti system, SP- Silvipasture system BP-Block plantation, Rd-R 


\begin{tabular}{|c|c|c|c|c|c|c|c|c|c|c|c|c|c|c|c|c|c|c|c|}
\hline \multirow[b]{2}{*}{ Sl.No } & \multirow[b]{2}{*}{ Scientific name } & \multicolumn{2}{|c|}{$\mathbf{B N D}(\mathrm{n}=31)$} & \multicolumn{2}{|c|}{ BDR(n=14) } & \multicolumn{2}{|c|}{$\operatorname{SCP}(n=3)$} & \multicolumn{2}{|c|}{ SH(n=9) } & \multicolumn{2}{|c|}{$\mathbf{S P}(n=2)$} & \multicolumn{2}{|c|}{$\mathbf{B N D}+\mathbf{B P}(\mathrm{n}=4)$} & \multicolumn{2}{|c|}{$\mathbf{B D R}+\mathbf{B P}(\mathrm{n}=2)$} & \multicolumn{2}{|c|}{$\mathbf{S H}+\mathbf{B P}(\mathrm{n}=6)$} & \multicolumn{2}{|c|}{$\mathbf{B N D + B D R}(\mathrm{n}=1)$} \\
\hline & & $\begin{array}{c}\text { Total } \\
\text { no. }\end{array}$ & $\mathrm{RD}$ & $\begin{array}{c}\text { Total } \\
\text { no. }\end{array}$ & $\mathrm{RD}$ & $\begin{array}{c}\text { Total } \\
\text { no. }\end{array}$ & $\mathrm{RD}$ & $\begin{array}{c}\text { Total } \\
\text { no. }\end{array}$ & $\mathrm{RD}$ & \begin{tabular}{|c|} 
Total \\
no.
\end{tabular} & $\mathrm{RD}$ & $\begin{array}{c}\text { Total } \\
\text { no. }\end{array}$ & $\mathrm{RD}$ & $\begin{array}{c}\text { Total } \\
\text { no. }\end{array}$ & $\mathrm{RD}$ & $\begin{array}{c}\text { Total } \\
\text { no. }\end{array}$ & $\mathrm{RD}$ & $\begin{array}{c}\text { Total } \\
\text { no. }\end{array}$ & $\mathrm{RD}$ \\
\hline 32 & Aegle marmelos & 1 & 0.03 & $\mathbf{0}$ & 0.00 & $\mathbf{0}$ & 0.00 & 1 & 0.09 & $\mathbf{0}$ & 0.00 & 2 & 0.50 & $\mathbf{0}$ & 0.00 & 1 & 0.11 & $\mathbf{0}$ & 0.00 \\
\hline 33 & Moringa oleifera & 1 & 0.03 & 8 & 0.54 & 4 & 1.92 & 3 & 0.27 & 1 & 1.04 & 1 & 0.25 & $\mathbf{0}$ & 0.00 & $\mathbf{0}$ & 0.00 & 2 & 1.17 \\
\hline 34 & Butea monosperma & 1 & 0.03 & $\mathbf{0}$ & 0.00 & $\mathbf{0}$ & 0.00 & $\mathbf{0}$ & 0.00 & $\mathbf{0}$ & 0.00 & 1 & 0.25 & $\mathbf{0}$ & 0.00 & $\mathbf{0}$ & 0.00 & $\mathbf{0}$ & 0.00 \\
\hline 35 & Sesbania grandiflora & 1 & 0.03 & $\mathbf{0}$ & 0.00 & 4 & 1.92 & $\mathbf{0}$ & 0.00 & $\mathbf{0}$ & 0.00 & $\mathbf{0}$ & 0.00 & $\mathbf{0}$ & 0.00 & $\mathbf{0}$ & 0.00 & $\mathbf{0}$ & 0.00 \\
\hline 36 & Pithecellobium dulce & 1 & 0.03 & 1 & 0.07 & $\mathbf{0}$ & 0.00 & $\mathbf{1}$ & 0.09 & $\mathbf{0}$ & 0.00 & $\mathbf{0}$ & 0.00 & 2 & 0.85 & 1 & 0.11 & 2 & 1.17 \\
\hline 37 & Ficus lacor & 1 & 0.03 & $\mathbf{0}$ & 0.00 & $\mathbf{0}$ & 0.00 & $\mathbf{0}$ & 0.00 & $\mathbf{0}$ & 0.00 & $\mathbf{0}$ & 0.00 & $\mathbf{0}$ & 0.00 & $\mathbf{0}$ & 0.00 & $\mathbf{0}$ & 0.00 \\
\hline 38 & Albizia procera & $\mathbf{0}$ & 0.00 & 3 & 0.20 & $\mathbf{0}$ & 0.00 & $\mathbf{0}$ & 0.00 & $\mathbf{0}$ & 0.00 & $\mathbf{0}$ & 0.00 & $\mathbf{0}$ & 0.00 & $\mathbf{0}$ & 0.00 & $\mathbf{0}$ & 0.00 \\
\hline 39 & Gliricidia sepium & $\mathbf{0}$ & 0.00 & 5 & 0.34 & $\mathbf{0}$ & 0.00 & $\mathbf{0}$ & 0.00 & $\mathbf{0}$ & 0.00 & $\mathbf{0}$ & 0.00 & 15 & 6.38 & $\mathbf{0}$ & 0.00 & $\mathbf{0}$ & 0.00 \\
\hline 40 & $\begin{array}{l}\text { Casuarina } \\
\text { equisetifolia }\end{array}$ & $\mathbf{0}$ & 0.00 & $\mathbf{0}$ & 0.00 & $\mathbf{0}$ & 0.00 & 5 & 0.45 & $\mathbf{0}$ & 0.00 & $\mathbf{0}$ & 0.00 & $\mathbf{0}$ & 0.00 & $\mathbf{0}$ & 0.00 & $\mathbf{0}$ & 0.00 \\
\hline 41 & Pongamia pinnata & $\mathbf{0}$ & 0.00 & 1 & 0.07 & $\mathbf{0}$ & 0.00 & 2 & 0.18 & $\mathbf{0}$ & 0.00 & $\mathbf{0}$ & 0.00 & 2 & 0.85 & $\mathbf{0}$ & 0.00 & $\mathbf{0}$ & 0.00 \\
\hline 42 & Ficus glomerata & $\mathbf{0}$ & 0.00 & 1 & 0.07 & $\mathbf{0}$ & 0.00 & 2 & 0.18 & 1 & 1.04 & $\mathbf{0}$ & 0.00 & 1 & 0.43 & 1 & 0.11 & $\mathbf{0}$ & 0.00 \\
\hline 43 & Borassus flabellifer & $\mathbf{0}$ & 0.00 & $\mathbf{0}$ & 0.00 & $\mathbf{0}$ & 0.00 & 1 & 0.09 & $\mathbf{0}$ & 0.00 & $\mathbf{0}$ & 0.00 & $\mathbf{0}$ & 0.00 & $\mathbf{0}$ & 0.00 & $\mathbf{0}$ & 0.00 \\
\hline 44 & Madhuca indica & $\mathbf{0}$ & 0.00 & 1 & 0.07 & $\mathbf{0}$ & 0.00 & $\mathbf{0}$ & 0.00 & 2 & 2.08 & $\mathbf{0}$ & 0.00 & $\mathbf{0}$ & 0.00 & $\mathbf{0}$ & 0.00 & $\mathbf{0}$ & 0.00 \\
\hline 45 & Acacia ferruginea & $\mathbf{0}$ & 0.00 & $\mathbf{0}$ & 0.00 & 1 & 0.48 & $\mathbf{0}$ & 0.00 & $\mathbf{0}$ & 0.00 & $\mathbf{0}$ & 0.00 & $\mathbf{0}$ & 0.00 & $\mathbf{0}$ & 0.00 & $\mathbf{0}$ & 0.00 \\
\hline 46 & $\begin{array}{l}\text { Acacia } \\
\text { auriculiformis }\end{array}$ & $\mathbf{0}$ & 0.00 & $\mathbf{0}$ & 0.00 & $\mathbf{0}$ & 0.00 & $\mathbf{0}$ & 0.00 & $\mathbf{0}$ & 0.00 & $\mathbf{0}$ & 0.00 & $\mathbf{0}$ & 0.00 & 1 & 0.11 & $\mathbf{0}$ & 0.00 \\
\hline 47 & Ailanthus excelsa & $\mathbf{0}$ & 0.00 & $\mathbf{0}$ & 0.00 & $\mathbf{0}$ & 0.00 & $\mathbf{1}$ & 0.09 & $\mathbf{0}$ & 0.00 & 1 & 0.25 & $\mathbf{0}$ & 0.00 & 1 & 0.11 & $\mathbf{0}$ & 0.00 \\
\hline 48 & Limonia acidissima & $\mathbf{0}$ & 0.00 & $\mathbf{0}$ & 0.00 & 1 & 0.48 & $\mathbf{0}$ & 0.00 & $\mathbf{0}$ & 0.00 & $\mathbf{0}$ & 0.00 & $\mathbf{0}$ & 0.00 & $\mathbf{0}$ & 0.00 & $\mathbf{0}$ & 0.00 \\
\hline 49 & Melia dubia & $\mathbf{0}$ & 0.00 & $\mathbf{0}$ & 0.00 & 20 & 9.62 & 45 & 4.07 & $\mathbf{0}$ & 0.00 & $\mathbf{0}$ & 0.00 & $\mathbf{0}$ & 0.00 & 55 & 5.93 & $\mathbf{0}$ & 0.00 \\
\hline 50 & Delonix regia & $\mathbf{0}$ & 0.00 & $\mathbf{0}$ & 0.00 & $\mathbf{0}$ & 0.00 & $\mathbf{0}$ & 0.00 & $\mathbf{0}$ & 0.00 & 2 & 0.50 & $\mathbf{0}$ & 0.00 & $\mathbf{0}$ & 0.00 & $\mathbf{0}$ & 0.00 \\
\hline 51 & Michelia champaca & $\mathbf{0}$ & 0.00 & $\mathbf{0}$ & 0.00 & $\mathbf{0}$ & 0.00 & 1 & 0.09 & $\mathbf{0}$ & 0.00 & $\mathbf{0}$ & 0.00 & $\mathbf{0}$ & 0.00 & 1 & 0.11 & $\mathbf{0}$ & 0.00 \\
\hline 52 & Muntingia calubura & $\mathbf{0}$ & 0.00 & $\mathbf{0}$ & 0.00 & $\mathbf{0}$ & 0.00 & $\mathbf{1}$ & 0.09 & $\mathbf{0}$ & 0.00 & $\mathbf{0}$ & 0.00 & $\mathbf{0}$ & 0.00 & $\mathbf{0}$ & 0.00 & $\mathbf{0}$ & 0.00 \\
\hline & $\begin{array}{l}\text { Total number of } \\
\text { trees }\end{array}$ & 2886 & & 1476 & & 208 & 0.00 & 1107 & & 96 & 0.00 & 399 & & 235 & & 927 & & 171 & \\
\hline & $\begin{array}{l}\text { Mean number of } \\
\text { trees ha-1 }^{-1}\end{array}$ & 93.10 & & 105.43 & & 69.33 & & 123.00 & & 48.00 & & 99.75 & & 117.50 & & 154.50 & & 171.00 & \\
\hline
\end{tabular}

RD- relative density and RF- Relative frequency 
Table.6 Dominant tree species found in different districts of north-eastern parts of Karnataka under irrigated ecosystem

\begin{tabular}{|c|c|c|c|c|c|c|c|c|c|c|c|c|c|}
\hline \multirow[t]{2}{*}{ Sl.No } & \multirow[t]{2}{*}{ Scientific Name } & \multicolumn{2}{|c|}{ Bidar $(n=12)$} & \multicolumn{2}{|c|}{ Kalaburagi $(n=12)$} & \multicolumn{2}{|c|}{\begin{tabular}{|l|} 
Yadgir $(n=12)$ \\
\end{tabular}} & \multicolumn{2}{|c|}{ Raichur (n=12) } & \multicolumn{2}{|c|}{ Ballari $(n=12)$} & \multicolumn{2}{|c|}{ Koppal $(n=12)$} \\
\hline & & Total no. & $\mathrm{RD}$ & Total no. & $\mathrm{RD}$ & Total no. & $\mathrm{RD}$ & Total no. & $\mathrm{RD}$ & Total no. & $\mathrm{RD}$ & Total no. & $\mathrm{RD}$ \\
\hline 1 & Tectona grandis & 817 & 72.62 & 842 & 67.74 & 970 & 73.65 & 610 & 59.34 & 659 & 52.14 & 829 & 54.25 \\
\hline 2 & Azadirachta indica & 85 & 7.56 & 143 & 11.50 & 111 & 8.43 & 100 & 9.73 & 106 & 8.39 & 185 & 12.11 \\
\hline 3 & Eucalyptus tereticornis & 36 & 3.20 & 17 & 1.37 & 1 & 0.08 & 6 & 0.58 & 0 & 0.00 & 13 & 0.85 \\
\hline 4 & Acacia nilotica & 35 & 3.11 & 36 & 2.90 & 22 & 1.67 & 0 & 0.00 & 2 & 0.16 & 10 & 0.65 \\
\hline 5 & Tamarindus indica & 26 & 2.31 & 22 & 1.77 & 5 & 0.38 & 7 & 0.68 & 40 & 3.16 & 7 & 0.46 \\
\hline 6 & Mangifera indica & 21 & 1.87 & 15 & 1.21 & 11 & 0.84 & 10 & 0.97 & 12 & 0.95 & 17 & 1.11 \\
\hline 7 & Annona squamosa & 21 & 1.87 & 25 & 2.01 & 8 & 0.61 & 19 & 1.85 & 2 & 0.16 & 0 & 0.00 \\
\hline 8 & Gliricidia sepium & 15 & 1.33 & 0 & 0.00 & 0 & 0.00 & 5 & 0.49 & 0 & 0.00 & 0 & 0.00 \\
\hline 9 & Syzygium cumini & 13 & 1.16 & 6 & 0.48 & 13 & 0.99 & 1 & 0.10 & 6 & 0.47 & 24 & 1.57 \\
\hline 10 & Psidium guajava & 8 & 0.71 & 10 & 0.80 & 8 & 0.61 & 4 & 0.39 & 7 & 0.55 & 4 & 0.26 \\
\hline 11 & Ziziphus mauritiana & 6 & 0.53 & 11 & 0.88 & 2 & 0.15 & 6 & 0.58 & 7 & 0.55 & 9 & 0.59 \\
\hline 12 & Cocos nucifera & 6 & 0.53 & 29 & 2.33 & 96 & 7.29 & 149 & 14.49 & 239 & 18.91 & 58 & 3.80 \\
\hline 13 & Leucaena leucocephala & 5 & 0.44 & 22 & 1.77 & 18 & 1.37 & 61 & 5.93 & 6 & 0.47 & 6 & 0.39 \\
\hline 14 & Santalum album & 4 & 0.36 & 9 & 0.72 & 6 & 0.46 & 2 & 0.19 & 5 & 0.40 & 1 & 0.07 \\
\hline 15 & Cassia fistula & 4 & 0.36 & 0 & 0.00 & 1 & 0.08 & 0 & 0.00 & 0 & 0.00 & 0 & 0.00 \\
\hline 16 & Achras sapota & 3 & 0.27 & 4 & 0.32 & 0 & 0.00 & 6 & 0.58 & 4 & 0.32 & 5 & 0.33 \\
\hline 17 & Phyllanthus emblica & 3 & 0.27 & 3 & 0.24 & 3 & 0.23 & 0 & 0.00 & 1 & 0.08 & 1 & 0.07 \\
\hline 18 & Hardwickia binata & 3 & 0.27 & 5 & 0.40 & 0 & 0.00 & 0 & 0.00 & 0 & 0.00 & 2 & 0.13 \\
\hline 19 & Bambusa arundinacea & 3 & 0.27 & 2 & 0.16 & 1 & 0.08 & 1 & 0.10 & 5 & 0.40 & 0 & 0.00 \\
\hline 20 & Albizia lebbek & 2 & 0.18 & 1 & 0.08 & 2 & 0.15 & 1 & 0.10 & 1 & 0.08 & 1 & 0.07 \\
\hline 21 & Citrus limon & 2 & 0.18 & 3 & 0.24 & 4 & 0.30 & 7 & 0.68 & 23 & 1.82 & 5 & 0.33 \\
\hline 22 & Murraya koenigii & 2 & 0.18 & 3 & 0.24 & 4 & 0.30 & 3 & 0.29 & 13 & 1.03 & 7 & 0.46 \\
\hline 23 & Melia azedarachta & 2 & 0.18 & 8 & 0.64 & 0 & 0.00 & 0 & 0.00 & 15 & 1.19 & 2 & 0.13 \\
\hline 24 & Aegle marmelos & 1 & 0.09 & 0 & 0.00 & 0 & 0.00 & 3 & 0.29 & 0 & 0.00 & 1 & 0.07 \\
\hline 25 & Butea monosperma & 1 & 0.09 & 0 & 0.00 & 1 & 0.08 & 0 & 0.00 & 0 & 0.00 & 0 & 0.00 \\
\hline 26 & Terminalia catappa & 1 & 0.09 & 3 & 0.24 & 6 & 0.46 & 1 & 0.10 & 0 & 0.00 & 1 & 0.07 \\
\hline 27 & Prosopis cineraria & 0 & 0.00 & 2 & 0.16 & 5 & 0.38 & 2 & 0.19 & 8 & 0.63 & 2 & 0.13 \\
\hline 28 & Albizia procera & 0 & 0.00 & 3 & 0.24 & 0 & 0.00 & 0 & 0.00 & 0 & 0.00 & 0 & 0.00 \\
\hline 29 & Grevillea robusta & 0 & 0.00 & 0 & 0.00 & 0 & 0.00 & 0 & 0.00 & 19 & 1.50 & 258 & 16.88 \\
\hline 30 & Casuarina equisetifolia & 0 & 0.00 & 0 & 0.00 & 5 & 0.38 & 0 & 0.00 & 0 & 0.00 & 0 & 0.00 \\
\hline 31 & Moringa oleifera & 0 & 0.00 & 5 & 0.40 & 5 & 0.38 & 6 & 0.58 & 2 & 0.16 & 2 & 0.13 \\
\hline
\end{tabular}




\begin{tabular}{|c|c|c|c|c|c|c|c|c|c|c|c|c|c|}
\hline \multirow[t]{2}{*}{ Sl.No } & \multirow[t]{2}{*}{ Scientific name } & \multicolumn{2}{|c|}{ Bidar $(n=12)$} & \multicolumn{2}{|c|}{$\begin{array}{l}\text { Kalaburagi } \\
(n=12)\end{array}$} & \multicolumn{2}{|l|}{$\begin{array}{l}\text { Yadgir } \\
(n=12)\end{array}$} & \multicolumn{2}{|c|}{$\begin{array}{l}\text { Raichur } \\
(n=12)\end{array}$} & \multicolumn{2}{|l|}{$\begin{array}{l}\text { Ballari } \\
(n=12)\end{array}$} & \multicolumn{2}{|l|}{$\begin{array}{l}\text { Koppal } \\
(n=12)\end{array}$} \\
\hline & & $\begin{array}{c}\text { Total } \\
\text { no. }\end{array}$ & $\mathrm{RD}$ & Total no. & $\mathrm{RD}$ & $\begin{array}{c}\text { Total } \\
\text { no. }\end{array}$ & $\mathrm{RD}$ & $\begin{array}{c}\text { Total } \\
\text { no. }\end{array}$ & $\mathrm{RD}$ & $\begin{array}{c}\text { Total } \\
\text { no. }\end{array}$ & $\mathrm{RD}$ & $\begin{array}{c}\text { Total } \\
\text { no. }\end{array}$ & $\mathrm{RD}$ \\
\hline 32 & Sesbania grandiflora & 0 & 0.00 & 1 & 0.08 & 0 & 0.00 & 0 & 0.00 & 4 & 0.32 & 0 & 0.00 \\
\hline 33 & Morinda pubescens & 0 & 0.00 & 3 & 0.24 & 0 & 0.00 & 2 & 0.19 & 6 & 0.47 & 0 & 0.00 \\
\hline 34 & Balanites roxburghii & 0 & 0.00 & 4 & 0.32 & 0 & 0.00 & 0 & 0.00 & 8 & 0.63 & 4 & 0.26 \\
\hline 35 & Pongamia pinnata & 0 & 0.00 & 1 & 0.08 & 2 & 0.15 & 2 & 0.19 & 0 & 0.00 & 0 & 0.00 \\
\hline 36 & Ficus glomerata & 0 & 0.00 & 3 & 0.24 & 1 & 0.08 & 1 & 0.10 & 0 & 0.00 & 1 & 0.07 \\
\hline 37 & Borassus flabellifer & 0 & 0.00 & 1 & 0.08 & 0 & 0.00 & 0 & 0.00 & 0 & 0.00 & 0 & 0.00 \\
\hline 38 & Madhuca indica & 0 & 0.00 & 1 & 0.08 & 0 & 0.00 & 0 & 0.00 & 2 & 0.16 & 0 & 0.00 \\
\hline 39 & Acacia ferruginea & 0 & 0.00 & 0 & 0.00 & 1 & 0.08 & 0 & 0.00 & 0 & 0.00 & 0 & 0.00 \\
\hline 40 & Pithecellobium dulce & 0 & 0.00 & 0 & 0.00 & 4 & 0.30 & 3 & 0.29 & 1 & 0.08 & 0 & 0.00 \\
\hline 41 & Acacia auriculiformis & 0 & 0.00 & 0 & 0.00 & 1 & 0.08 & 0 & 0.00 & 0 & 0.00 & 0 & 0.00 \\
\hline 42 & Ficus religiosa & 0 & 0.00 & 0 & 0.00 & 0 & 0.00 & 2 & 0.19 & 0 & 0.00 & 0 & 0.00 \\
\hline 43 & Chloroxylon swietenia & 0 & 0.00 & 0 & 0.00 & 0 & 0.00 & 3 & 0.29 & 0 & 0.00 & 2 & 0.13 \\
\hline 44 & Wrightia tinctoria & 0 & 0.00 & 0 & 0.00 & 0 & 0.00 & 4 & 0.39 & 0 & 0.00 & 0 & 0.00 \\
\hline 45 & Ailanthus excelsa & 0 & 0.00 & 0 & 0.00 & 0 & 0.00 & 1 & 0.10 & 1 & 0.08 & 1 & 0.07 \\
\hline 46 & Phoenixa dactylifera & 0 & 0.00 & 0 & 0.00 & 0 & 0.00 & 0 & 0.00 & 1 & 0.08 & 2 & 0.13 \\
\hline 47 & Limonia acidissima & 0 & 0.00 & 0 & 0.00 & 0 & 0.00 & 0 & 0.00 & 1 & 0.08 & 0 & 0.00 \\
\hline 48 & Melia dubia & 0 & 0.00 & 0 & 0.00 & 0 & 0.00 & 0 & 0.00 & 55 & 4.35 & 65 & 4.25 \\
\hline 49 & Delonix regia & 0 & 0.00 & 0 & 0.00 & 0 & 0.00 & 0 & 0.00 & 2 & 0.16 & 0 & 0.00 \\
\hline 50 & Michelia champaca & 0 & 0.00 & 0 & 0.00 & 0 & 0.00 & 0 & 0.00 & 1 & 0.08 & 1 & 0.07 \\
\hline 51 & Muntingia calubura & 0 & 0.00 & 0 & 0.00 & 0 & 0.00 & 0 & 0.00 & 0 & 0.00 & 1 & 0.07 \\
\hline \multirow[t]{3}{*}{52} & Ficus lacor & 0 & 0.00 & 0 & 0.00 & 0 & 0.00 & 0 & 0.00 & 0 & 0.00 & 1 & 0.07 \\
\hline & $\begin{array}{l}\text { Total number of } \\
\text { trees }\end{array}$ & 1125 & 0.00 & 1243 & & 1317 & & 1028 & & 1264 & & 1528 & \\
\hline & $\begin{array}{l}\text { Mean number of } \\
\text { trees ha }^{-1}\end{array}$ & 93.75 & $\mathbf{0 . 0 0}$ & 103.58 & & 109.75 & & 85.67 & & 105.33 & & $\mathbf{1 2 7 . 3 3}$ & \\
\hline
\end{tabular}

Note: RD- Relative Density 
Fig.1 Shannon's index for no. of trees with farm holding size under irrigated ecosystem

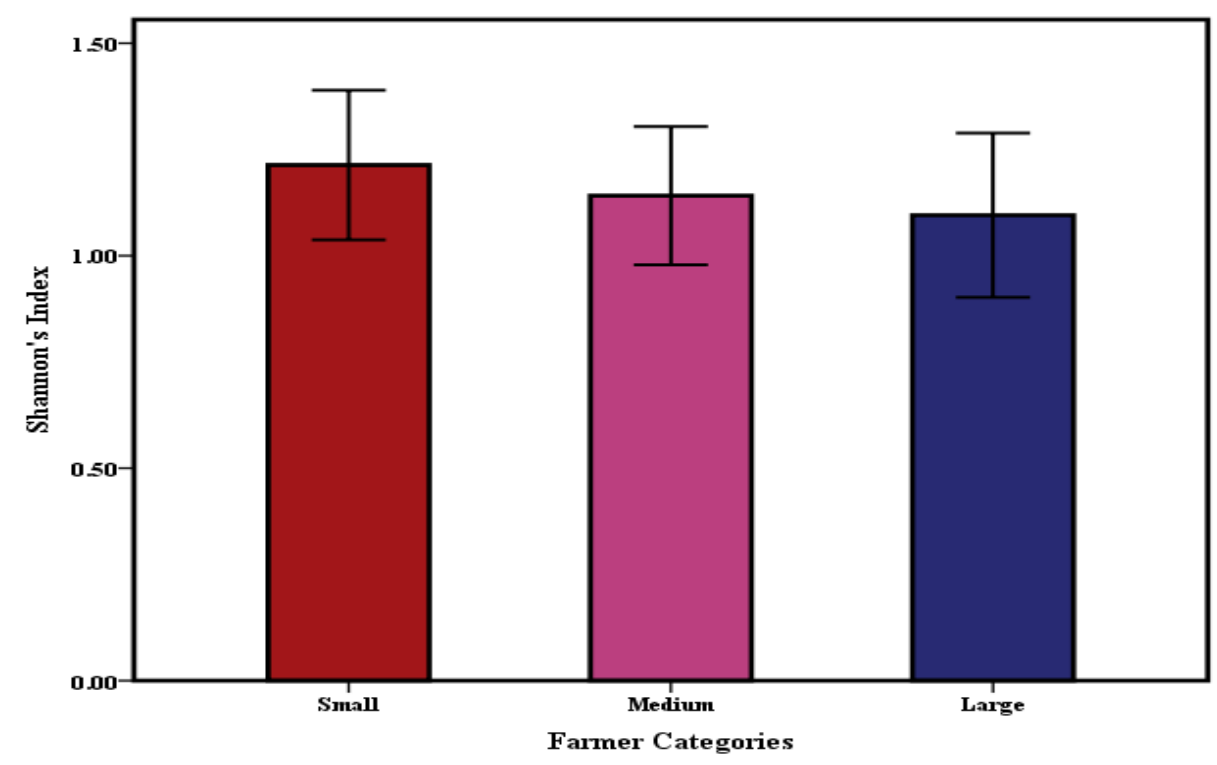

Fig.2 Species density in different districts under irrigated ecosystem

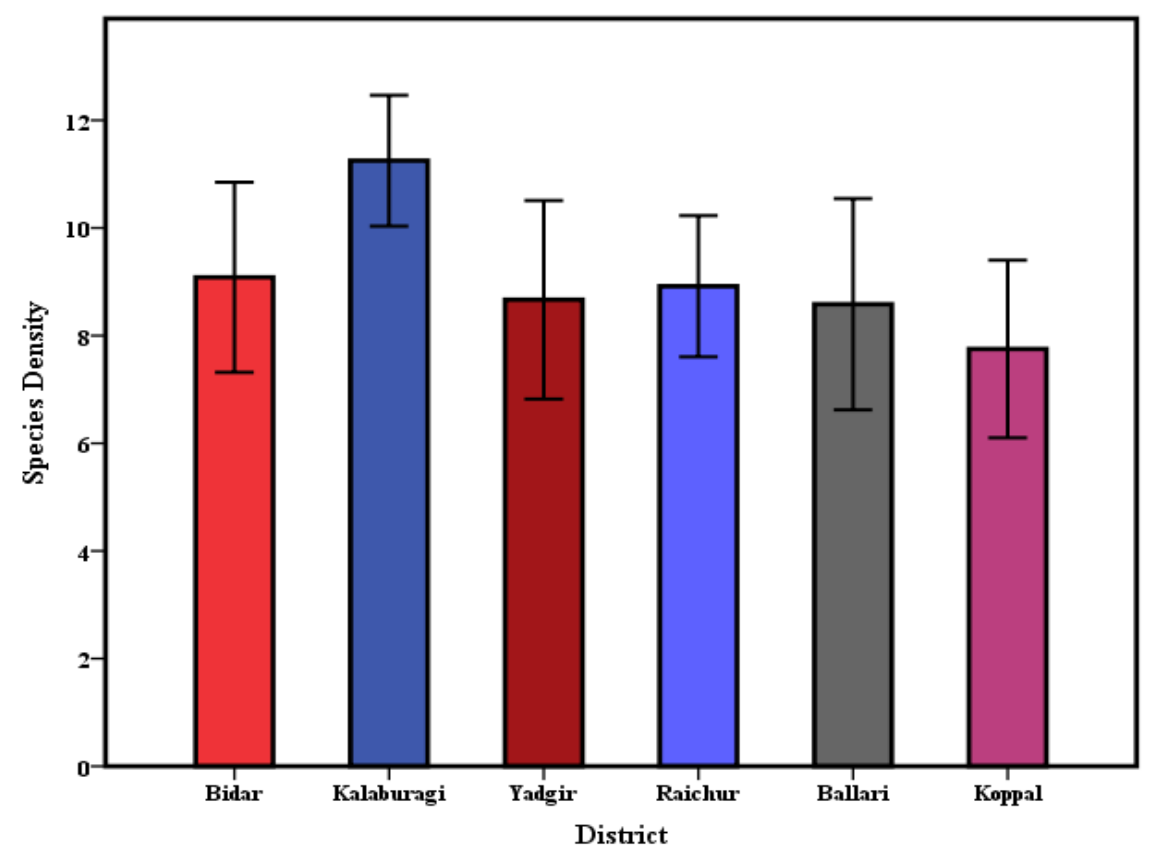

On the contrary lower species density was recorded in Koppal and Bllari which may be attributed to lower rainfall in this region and the major agroforestry systems practiced were silvihorti and bund planting which contributed for the lower density of species. In silvihorti system and bund planting farmers grow only few economically important species unlike boundary and scattered planting. This indicated that certain species are preferred in specific agroforestry systems. The results are in line with Nyaga et al., (2015) who also reported preference for certain species in certain agroforestry systems. Henry et al., 
(2009) reported higher diversity in homestead and farm land where different trees distributed were proportional, and lower diversity was observed in wood lot and hedge planting.

\section{Species dominance in agroforestry systems}

A total of 7525 individuals of 52 species were recorded. Tectona grandis $(\mathrm{n}=4817$ $\mathrm{RF}=94.44$ ) was the most predominant species found in the irrigated ecosystem followed by Azadirachta indica $(\mathrm{n}=660, \mathrm{RF}=100.0)$ and Cocos nucifera $(\mathrm{n}=577, \mathrm{RF}=55.56)$ while 23 other species recorded less than 10 individuals (Table 4).

Among the categories of the farmers, Tectona grandis was found to be the dominant species followed by Azadirachta indica and Cocos nucifera with all the categories of farmers (Table 4). The dominance of the species was worked out with respect to the different agroforestry systems (Table 5). It was found that Tectona grandis, Azadirachta indica and Cocus nucifera were the dominant tree species among the agroforestry systems. However, considerable variation in the order of the dominance of species was observed. Tectona grandis followed by Azadirachta indica and Cocos nucifera were the order dominance in bund and boundary planting whereas Cocos nucifera followed by Azadirachta indica and Tectona grandis was the preferential order in scattered planting, Tectona grandis followed by Cocus nucifera and Azadirachta indica in in silvihorti system, and in silvipastoral system it was Azadirachta indica followed by Cocos nucifera and Tectona grandis was the order of preference.

Among the districts, Tectona grandis was the dominant tree species (Table 6). However, considerable variations were noticed between the districts with the respect to the dominance of the other species. Tectona grandis followed by Azadirachta indica and Eucalyptus tereticornis were dominant in Bidar district, and Tectona grandis, Azadirachta indica and Acacia nilotica were the dominant species in Kalaburagi district. In Yadgir and Raichur districts Tectona grandis followed by Azadirachta indica and Cocos nucifera were dominating species. The dominant species in Bellary district were Tectona grandis, Cocos nucifera and Azadirachta indica. In Koppal district Tectona grandis, Azadirachta indica and Grevillea robusta were the dominant ones.

In all, the findings indicated that Tectona grandis, Azadirachta indica and Cocos nucifera were the predominant species found in the agroforestry system under irrigated ecosystem. This gives an insight that farmers preferably and selectively grow these trees on the farm mainly for economical reasons, multipurpose utility and personal/cultural preference of the locality. Though Tectona grandis is not indigenous to this region farmer preferred this species mainly for its high economic value, availability of market, and knowledge about the growth habit of the species as teak is deciduous in nature, grows straight with shallow crown. The other dominant species were Azadirachta indica and Cocos nucifera. Azadirachta indica is a multipurpose indigenous species and Cocos nucifera gives substantial/regular additional income to the irrigated farmers. The farmer also grew exotic species like eucalyptus and silver oak which are short rotation species. This indicates that farmers have the knowledge about these species, hence grew them in short rotation to get income at short interval. Although, 85 per cent of total tree species were indigenous to the area farmer grew exotics and economical valuable species in higher density. The results are in line with the earlier study Bucagu et al., (2013) who in their study on assessment of farmers interest on agroforestry systems in Simbi region of 
Rwanda recorded that Grevillea robusta and Eucalyptus urophyla were predominantly integrated in the agroforestry systems by the farmers for their economical value and fast growth rate; preferably Grevillea for the higher biomass it produces in short rotation and it is less competitive with field crops.

The study recorded six major agroforestry systems practiced by the farmers under irrigated ecosystem. However bund and boundary planting were the most preferred agroforestry systems in the region. The farm holding size and ecological condition of the region influenced the kind of agroforestry systems practiced. Large farmers preferred silvihorti system followed by bund and boundary planting whereas small and medium farmers preferred bund planting followed boundary planting. Further, variation in the kind of agroforestry followed was also observed with different locations. Overall, the practice of agroforestry systems found to be greatly influenced by the socioeconomic condition of the farmer and ecological condition of the area. The study recorded 52 tree species with mean species density of 9.04 $\mathrm{ha}^{-1}$ and mean number of 104.24 trees $\mathrm{ha}^{-1}$. The Number of trees per hectare was positively correlated with size of land holding and kind of agroforestry systems whereas the species richness was found to be positively correlated with the ecological conditions of the locations.

However, the higher Shannon's diversity indices were recorded in small farmers followed by medium and large farmers which indicated the equi distribution of useful tree species in small land holding than in the large land holding. Finally, agroforestry land use system was found quite complex, and the structure and composition are greatly influenced by the heterogeneous factors including socio economic status of the farmer and ecological conditions of the area.

\section{References}

Abebe, T., Sterck, F. J., Wiersum, K. F. and Bongers, F., 2013, Diversity, composition and density of trees and shrubs in agroforestry homegardens in Southern Ethiopia. Agroforestry Systems, 87:1283-1293.

Behera, M. C., and Dhir, B. C., 2013, Phytosociological study of woody components in traditionalagro-forestry systems of Boudh district, Odisha, India. International Journal of Farm Sciences, 3(2):63-76.

Bellow, J.G., Hudson, R.F. and Nair, P.K.R., 2008, Adoption of fruit-tree-based agroforestry on small farms in the subtropical highlands. Agroforest. Syst., 73:23-36.

Bucagu, C., Vanlauwe, B., Van Wijk, M.. T. and Giller, K. E., 2013, Assessing farmer's interest in agroforestry in two contrasting agro-ecological zones of Rwanda. Agroforestry Systems, 87:141158.

Christine, O., and Nestor S., 2008, Traditional agroforestry systems as tools for conservationBof genetic resources of Milicia excelsa Welw. C.C. Bergin Benin. Agroforestry Systems, 74:17-26.

Giller, K.E., Rowe, E.C., DE-Ridder, N. and Van-Keulen, H., 2006, Resource use dynamics and interaction in tropics: scaling up in space and time. Agroforest. Syst., 88: 8-27.

Henry, M., Tittonell, P., Manlay, R. J., Bernoux, M., Albrecht, A. and Vanlauwe, B., 2009, Biodiversity, carbon stock and sequestration potential in above ground biomass in smallholder farming systems of western Kenya. Agriculture, Ecosystems and Environment, 129:238-252.

Nair, P.K.R., 2011, Agroforestry systems and environmental quality: Introduction. Journal environmental Quality 40:784- 
790

Nair, P.K.R., Gordon, A. M. and MosqueraLosada, M. R., 2008, Agroforestry. In: Jorgenson SE, Fath BD (eds) Ecological engineering: encyclopedia of ecology, vol 1, pp 101-110.Elsevier, Oxford.

Nerlich, K., Graeff-Honninger, S. and Claupein, W., 2013, Agroforestry in Europe: a review of the disappearance of traditional systems and development of modern agroforestry practices, with emphasis on experiences in Germany. Agroforestry Systems, 87 (2): 475-492.

Nyaga, J., Barrios, E., Muthuri, C.W., Oborn,
I., Matiru, V. and Sinlair, F.L., 2015, Evaluating factors influencing heterogeneity in agroforestry adoption and practices within smallholder farmers in Rift Valley, Kenya. Agriculture, Ecosystems and Environment, 212:106-118.

Tittonell, P., Vanlauwe, B., Leffelaar, P.A., Shepherd, K.D. and Giller, K.E., 2005, Exploring diversity in soil fertility management of small holder farms in western Kenya. I. Heterogeneity at region and farm scale. Agro. Ecosyst. Environ, 110:149-165.

\section{How to cite this article:}

Doddabasawa, M. Mahadeva Murthy and Chittapur, B.M. 2017. Assessment of Tree Diversity in Agroforestry Systems under Irrigated Ecosystem. Int.J.Curr.Microbiol.App.Sci. 6(10): 31113127. doi: https://doi.org/10.20546/ijcmas.2017.610.366 